Ann. Biol. anim. Bioch. Biophys., 1979, 19 (1B), 217-223.

\title{
Conséquences d'une très forte restriction azotée à différentes périodes de la gestation de la truie sur le développement des fœtus
}

\author{
par $M$. LEUILLET, M. ETIENNE *, E. SALMON-LEGAGNEUR ** \\ avec la collaboration technique de Chantal BLONDEL, J. C. LE MENTEC \\ I.T.C.F., 8 avenue du Président Wilson, 75116 Paris. \\ * Station de Recherches sur l'Elevage des Porcs, I.N.R.A., 78350 jouy en josas. \\ ** INRA, C.R.A.A.G., Petit Bourg 97170 Guadeloupe.
}

Summary. Feeding a very protein-poor diet to sow at different periods during pregnancy. Effects on fefal development.

We report an experiment using 51 multiparous sows to determine the effects of protein nutrition on the litter during pregnancy. Two diets were offered to the animals; one was a protein-poor ration containing 1.3 p. 100 protein $(p)$ and the other was a control diet containing 10.6 p. 100 protein (P). The sows were divided into lots according to dief distribution sequence during pregnancy and were fed $2.5 \mathrm{~kg}$ daily of either one diet or the other. No significant difference was noted in fefus number or weight in the different groups 60 days after service. However, at farrowing the mean weight of the piglets receiving dief $p$ was lower as that diet was given over a longer period. When it was provided during the whole pregnancy, the development of uterine contents slowed down : the dry matter level in placenta and piglet body and the protein percentage of piglet dry matter decreased. The weight of several organs (liver, heart, kidneys, adrenals) and the femur length were reduced almost in proportion to total animal weight. Only brain weight was unaffected. These piglets were observed to be weaker between birth and weaning. On the contrary, the body development of offspring from sows fed the control diet during at least 25 days at the beginning or end of pregnancy seemed to be much less affected. These results confirm both the low protein requirements of the sow during pregnancy and that the mother's body acts as a buffer against nutrient deficiencies.

\section{Introduction.}

L'influence de la nutrition azotée pendant la gestation sur les performances de reproduction de la truie est en général faible (Salmon-Legagneur, 1964 ; Duée et Sève, 1978). La prolificité est le plus souvent inchangée, et seul le poids moyen des porcelets se trouve parfois diminué à la naissance dans le cas d'une restriction protéique sévère. Ainsi, Rippel et al. (1965) n'observent aucune différence sur la portée de truies recevant un régime à 5 ou 16 p. 100 de matières azotées pendant les 50 derniers jours précédant la mise bas. Pond et al. (1968) montrent que la gestation peut se 
dérouler, même pour des truies n’ingérant quotidiennement que $9 \mathrm{~g}$ de protéines entre la saillie et la parturition. De Geeter et al. (1972), puis Hammell et al. (1976), aboutissent à la même conclusion sur des effectifs importants d'animaux. Toutefois, Pond et al. (1969) montrent qu'un apport temporaire de protéines au moment de l'implantation des blastocystes (16e au $20^{\mathrm{e}}$ jour post saillie) a un effet bénéfique sur le développement des fœetus, le poids des porcelets à la naissance et leur vitesse de croissance jusqu'à $90 \mathrm{~kg}$. Ceci met donc l'accent sur des besoins différentiels suivant le stade de gestation, et il nous a paru intéressant de préciser les effets de différentes séquences de distribution d'un aliment protéiprive à des truies gravides sur le développement fœal.

\section{Matériel et méthodes.}

L'expérience porte sur 51 truies multipares de race Large White, d'un poids moyen de $170 \mathrm{~kg}$, réparties en 4 lots après la saillie suivant la séquence de distribution des aliments témoin $(P)$ ou à très faible teneur en protéines $(P)$ dont la composition est rapportée au tableau 1. Les femelles du lot PP reçoivent l'aliment $P$ pendant toute la

\section{TABLEAU 1}

Composition des aliments (p. 100)

\begin{tabular}{|c|c|c|}
\hline Régime & $\mathbf{P}$ & $\mathbf{P}$ \\
\hline 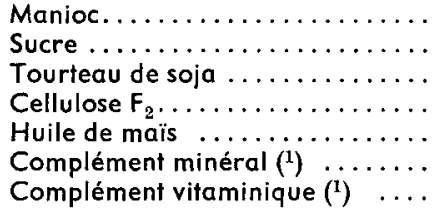 & $\begin{array}{l}62,6 \\
20,0 \\
\overline{9}, 0 \\
3,0 \\
5,0 \\
0,4\end{array}$ & $\begin{array}{r}44,6 \\
20,0 \\
20,0 \\
7,0 \\
3,0 \\
5,0 \\
0,4\end{array}$ \\
\hline & 100,0 & 100,0 \\
\hline Protéines p. $100(\mathrm{~N} \succ .6,25) \ldots \ldots$ & 1,3 & 10,6 \\
\hline
\end{tabular}
(1973).

(1) Compléments minéral et vitaminique dont la composition est rapportée par Etienne et Henry

gestation, et celles du lot PP, le régime p durant la même période. Les animaux des autres lots consomment l'aliment $P$ pendant 90 jours, soit à la fin (lot Pp), soit au début de la gestation (lot $p P$ ), le régime témoin étant distributé au cours des 25 autres jours. Le niveau de consommation de $2,5 \mathrm{~kg} /$ jour permet une ingestion quotidienne de 32 et $265 \mathrm{~g}$ de protéines respectivement pour les aliments $p$ et $P$. Pendant la lactation de 5 semaines, les truies reçoivent à volonté un aliment à 15,5 p. 100 de matières azotées. Cinq truies des lots PP, PP et Pp sont abattus à 60 jours de gestation, ef 9 femelles dans chaque lot mettent bas. Outre l'enregistrement des performances de reproduction, diverses déterminations sont effectuées sur le placenta et la portée à 60 jours après la fécondation et à la naissance : dénombrement des fœtus, poids total et, sur quelquesuns d'entre eux, poids de certains organes, analyse chimique globale (teneur en 
matière sèche, protéines, lipides, matières minérales), et dosage du glycogène sur le foie et la carcasse des fœetus, et le foie du porcelet. Les résultats sont comparés par le test de Newman-Keuls.

TABLEAU 2

Résultats de reproduction ( $\left.{ }^{1}\right)$

\begin{tabular}{|c|c|c|c|c|c|}
\hline Lot & PP & PP & $P_{p}$ & $p P$ & $\begin{array}{l}\mathrm{CV}\left({ }^{2}\right) \\
\text { P. } 100\end{array}$ \\
\hline $\begin{array}{l}\text { Nombre de truies mettant bas } \ldots \ldots \ldots \ldots \ldots \ldots \\
\text { Nombre total de porcelets nés/portée } \ldots \ldots \ldots \ldots \\
\text { Nombre de porcelets nés vivants/portée } \ldots \ldots \ldots \ldots \\
\text { Poids moyen du porcelet né vivant }(\mathrm{kg}) \ldots \ldots \ldots \ldots \\
\text { Poids de la portée à la naissance }(\mathrm{kg}) \ldots \ldots \ldots \ldots \\
\text { Poids moyen du porcelet à } 35 \text { jours }(\mathrm{kg}) \ldots \ldots \ldots \ldots \\
\text { Nombre de portées sevrées } \ldots \ldots \ldots \ldots \ldots \ldots\end{array}$ & $\begin{array}{l}9 \\
12,3 \\
10,2 \\
1,24_{\mathrm{a}} \\
12,7_{\mathrm{a}} \\
8,63_{\mathrm{a}} \\
9\end{array}$ & $\begin{array}{l}8 \\
9,9 \\
7,1 \\
0,95_{b} \\
6,4_{b} \\
5,59_{b} \\
6\end{array}$ & $\begin{array}{l}9 \\
10,6 \\
8,8 \\
1,09_{\mathrm{ab}} \\
8,8_{\mathrm{b}} \\
7,18_{\mathrm{a}} \\
\mathbf{8}\end{array}$ & $\begin{array}{l}9 \\
9,6 \\
8,1 \\
1,04_{a b} \\
8,3_{b} \\
7,72_{a} \\
9\end{array}$ & $\begin{array}{l}\overline{28,7} \\
39,4 \\
18,9 \\
35,9 \\
18,8\end{array}$ \\
\hline
\end{tabular}

(1) Les résultats d'une même ligne indexés par la même lettre ne diffèrent pas significativement.

(2) CV p. 100 : Coefficient de variation p. 100.

TABLEAU 3

Résultats de dissection ef d'analyse à l'abattage à 60 jours de gestation (5 truies/lot)

\begin{tabular}{|c|c|c|c|c|}
\hline Lot & PP & $\mathrm{PP}$ & $P_{p}$ & $\begin{array}{c}C V \\
\text { p. } 100\end{array}$ \\
\hline 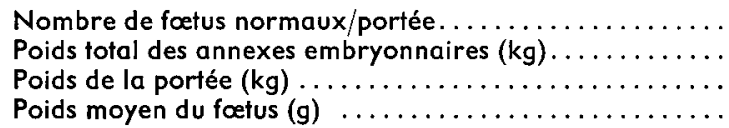 & $\begin{array}{c}8,4 \\
1,61 \\
1,13 \\
134\end{array}$ & $\begin{array}{l}8,8 \\
1,70 \\
1,02 \\
116\end{array}$ & $\begin{array}{c}8,8 \\
1,45 \\
0,92 \\
100\end{array}$ & $\begin{array}{l}40,9 \\
40,4 \\
36,0 \\
21,2\end{array}$ \\
\hline \multicolumn{5}{|l|}{ Placenta } \\
\hline Matière sèche (p. 100) & 4,77 & 4,79 & 5,36 & 13,8 \\
\hline \multicolumn{5}{|l|}{ En p. 100 de la matière sèche : } \\
\hline 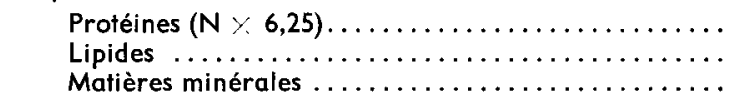 & $\begin{array}{r}63,0 \\
7,8 \\
18,1\end{array}$ & $\begin{array}{r}62,6 \\
9,1 \\
18,1\end{array}$ & $\begin{array}{r}63,0 \\
8,4 \\
16,8\end{array}$ & $\begin{array}{r}5,4 \\
29,8 \\
14,4\end{array}$ \\
\hline
\end{tabular}

\section{Fofus}

Poids du foie (g) $\ldots \ldots \ldots \ldots \ldots \ldots \ldots \ldots \ldots \ldots \ldots \ldots \ldots \ldots \ldots \ldots, 6,68$

Poids des 2 reins $(g) \quad \ldots \ldots \ldots \ldots \ldots \ldots \ldots \ldots \ldots \ldots$

Longueur du fémur $(\mathrm{mm}) \ldots \ldots \ldots \ldots \ldots \ldots \ldots \ldots$

Matière sèche $(p .100) \ldots \ldots \ldots \ldots \ldots \ldots \ldots \ldots \ldots \ldots$.

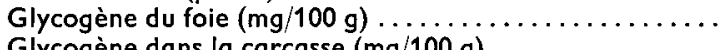

Glycogène dans la carcasse $(\mathrm{mg} / 100 \mathrm{~g}) \ldots \ldots \ldots \ldots \ldots$

En p. 100 de la matière sèche :

Protéines $(\mathrm{N} \times 6,25) \ldots \ldots \ldots \ldots \ldots \ldots \ldots \ldots \ldots$

Lipides ...............................

Matières minérales
1,49

23

$\begin{array}{cc}23 & 21 \\ 9,38 & 9,50 \\ 454 & 343 \\ 746 & 771\end{array}$

$\begin{array}{cr}5,45 & 28,2 \\ 1,21 & 27,0 \\ 21 & 17,7 \\ 9,44 & 4,7 \\ 335 & 71,8 \\ 755 & 11,2\end{array}$

63,0

8,8

20,7

62,6
8,5
21,3

63,0

8,5

21,1 


\section{Résultats.}

Il n'apparaît pas de différence significative sur le nombre de portées nées, ni sur la prolificité (tabl. 2). Le poids moyen à la naissance des porcelets du lot pp est significativement plus faible que celui des animaux du lot PP, la valeur étant intermédiaire dans les 2 autres groupes. Les mêmes résultats se retrouvent au sevrage, mais le nombre de portées qui parviennent à ce stade est réduit dans le lot pp.

A 60 jours de gestation (tabl. 3), aucune différence significative n'est observée sur la taille de la portée, le poids des annexes embryonnaires et des fœtus. De même, la composition chimique du placenta et des fœetus est rigoureusement comparable dans les 3 lots. Le poids du foie et des reins, ef la longueur du fémur des fotus, ne sont pas affectés par le régime.

Les résultats des déterminations effectuées à la naissance sont présentés au tableau 4. Si le poids du placenta n'est pas modifié, sa teneur en matière sèche diminue,

TABLEAU 4

Résultats de dissection ef d'analyse à la naissance

\begin{tabular}{|c|c|c|c|c|c|}
\hline Lot & PP & PP & $\mathrm{Pp}$ & $\mathrm{pP}$ & $\begin{array}{l}\text { CV } \\
\text { p. } 100\end{array}$ \\
\hline \multicolumn{6}{|l|}{ Placenta } \\
\hline $\begin{array}{l}\text { Poids }(\mathrm{kg}) \ldots \ldots \ldots \ldots \ldots \ldots \ldots \ldots \ldots \ldots \\
\text { Matière sèche }(\text { p. 100) } \ldots \ldots \ldots \ldots \ldots \ldots \ldots\end{array}$ & $\begin{array}{l}1,93 \\
9,26\end{array}$ & $\begin{array}{l}1,94 \\
6,18_{b}\end{array}$ & $\begin{array}{l}2,07 \\
6,87_{b}\end{array}$ & $\begin{array}{l}1,90 \\
7,37_{b}\end{array}$ & $\begin{array}{l}33,9 \\
19,8\end{array}$ \\
\hline \multicolumn{6}{|l|}{ En p. 100 de la matière sèche : } \\
\hline $\begin{array}{l}\text { Protéines }(\mathbf{N} \times \mathbf{6 , 2 5}) \ldots \ldots \ldots \ldots \ldots \ldots \ldots \\
\text { Lipides } \quad \ldots \ldots \ldots \ldots \ldots \ldots \ldots \ldots \ldots \\
\text { M. minérales } \ldots \ldots \ldots \ldots \ldots \ldots \ldots \ldots\end{array}$ & $\begin{array}{r}68,8 \\
6,6 \\
11,6\end{array}$ & $\begin{array}{r}67,9 \\
7,8 \\
15,9 b\end{array}$ & $\begin{array}{r}65,5 \\
7,9 \\
14,9_{b}\end{array}$ & $\begin{array}{r}67,4 \\
6,8 \\
13,4 \mathrm{ab}\end{array}$ & $\begin{array}{r}4,7 \\
15,5 \\
19,9\end{array}$ \\
\hline \multicolumn{6}{|l|}{ Porcelets } \\
\hline $\begin{array}{l}\text { Nombre de porcelets analysés } \ldots \ldots \ldots \ldots \ldots \ldots \\
\text { Poids moyen des porcelets analysés, } g \ldots \ldots \ldots\end{array}$ & $\begin{array}{r}14 \\
1214\end{array}$ & $\begin{array}{r}14 \\
754\end{array}$ & $\begin{array}{r}17 \\
911\end{array}$ & $\begin{array}{r}16 \\
937\end{array}$ & $\overline{40,8}$ \\
\hline \multicolumn{6}{|l|}{ Résultats de dissection : } \\
\hline 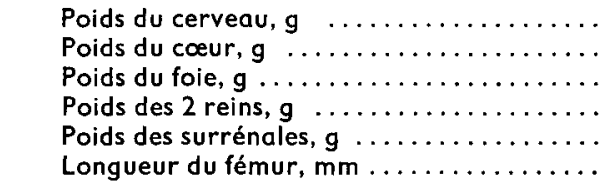 & $\begin{array}{r}29,5 \\
8,3 \\
31,2 \\
8,2 \mathrm{a} \\
0,33 \\
66_{\mathrm{a}}\end{array}$ & $\begin{array}{c}29,0 \\
6,9 \\
21,0 \\
5,8_{b} \\
0,25 \\
47_{b}\end{array}$ & $\begin{array}{r}28,6 \\
7,0 \\
25,2 \\
6,7_{\mathrm{b}} \\
0,29 \\
52_{\mathrm{ab}}\end{array}$ & $\begin{array}{r}29,8 \\
7,4 \\
25,4 \\
6,1_{b} \\
0,29 \\
53_{\mathrm{ab}}\end{array}$ & $\begin{array}{r}9,3 \\
37,2 \\
47,3 \\
38,1 \\
41,3 \\
35,1\end{array}$ \\
\hline \multicolumn{6}{|l|}{ Résultats d'analyse : } \\
\hline M. sèche (p. 100) & $18,8_{\mathrm{a}}$ & $15,8_{\mathrm{b}}$ & $17,6 a$ & $18,2_{\mathrm{a}}$ & 7,7 \\
\hline \multicolumn{6}{|l|}{ en p. 100 de la m. sèche : } \\
\hline 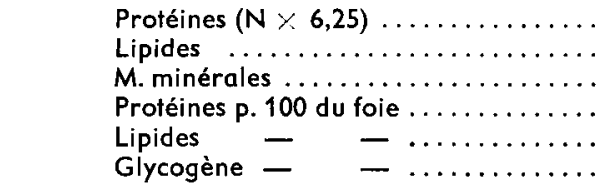 & $\begin{array}{c}61,0_{\mathrm{a}} \\
8,0 \\
20,7 \\
14,7_{\mathrm{a}} \\
3,2_{\mathrm{a}} \\
4,5_{\mathrm{a}}\end{array}$ & $\begin{array}{c}54,9_{\mathrm{b}} \\
8,1 \\
21,1 \\
12,1_{\mathrm{b}} \\
2,4_{\mathrm{b}} \\
9,4_{\mathrm{b}}\end{array}$ & $\begin{array}{c}59,1_{\mathrm{a}} \\
8,9 \\
20,8 \\
14,8_{\mathrm{a}} \\
2,9_{\mathrm{ab}} \\
3,0_{\mathrm{a}}\end{array}$ & $\begin{array}{c}60,0_{\mathrm{a}} \\
9,5 \\
21,3 \\
15,4_{\mathrm{a}} \\
3,0_{\mathrm{ab}} \\
2,8_{\mathrm{a}}\end{array}$ & $\begin{array}{r}6,3 \\
16,9 \\
8,4 \\
13,2 \\
15,1 \\
8,1\end{array}$ \\
\hline Protéines dans le contenu utérin total (g) & $1886_{\mathrm{s}}$ & $932_{b}$ & $1118_{\mathrm{bc}}$ & $1351_{c}$ & 20,8 \\
\hline
\end{tabular}


et le taux de minéraux dans cette dernière s'accroît lorsque les truies ont consommé l'aliment $p$ à un moment donné de la gestation.

En ce qui concerne les porcelets, le poids du cerveau est le même dans les 4 lots. Par contre, le poids des autres organes (cœur, foie, surrénales) tend à diminuer chez les animaux issus des 3 lots protéiprives, l'écart étant significatif pour le poids des reins ef la longueur du fémur. On peut d'ailleurs noter que pour chacune de ces mesures, les valeurs observées dans les lois $P p$ et $P P$ sont intermédiaires. De la même façon, la teneur en matière sèche et le taux de protéines dans cette dernière sont significativement plus faibles chez les porcelets du lot pp que chez les autres, les proportions de lipides et de minéraux n'étant pas affectées. Le foie des porcelets issus de mères PP a une teneur également moindre en protéines et en lipides, mais supérieure en glycogène. Cependant, les données de composition chimique chez les animaux des lots $P p$ et PP ne se distinguent pas de celles obtenues dans le lot témoin PP. Mais compte tenu du poids de la portée qui tend à décroître dans les 3 lołs protéiprives, la quantité de matières azotées présentes dans les contenus utérins (fœtus + annexes embryonnaires + liquides) va en se réduisant du lot PP aux lots $\mathrm{PP}, \mathrm{Pp}$ et $\mathrm{pP}$.

\section{Discussion.}

Une carence importante en protéines n'affecte pas significativement la prolificité. Cependant, le nombre de fotus ou de porcelets nés totaux ou nés vivants tend à diminuer dans les 3 lots de truies recevant le régime $p$ pendant tout ou partie de la gestation. La même remarque avait été faite dans des conditions analogues par Pond et al. (1968), De Geeter et al. (1972), Atinmo ef al. (1974a) ou Hammel et al. (1976). Par contre, l'effet de la carence azotée sur le poids moyen du porcelet est significatif, et d'autant plus marqué que la distribution du régime à faible teneur en protéines est effectuée plus longtemps. Une réduction pondérale de près de 20 p. 100 a été observée à la suite de la consommation d'un aliment déficient en lysine par les truies (Duée et Rérat, 1975). Pond et al. (1969) suggèrent que par rapport à un régime protéiprive, la distribution d'un régime équilibré au moment de l'implantation des blastocystes peut avoir un effet favorable sur leur développement ultérieur. Cependant, l'amélioration est davantage marquée lorsque les truies reçoivent cet aliment pendant les 24 premiers jours de gestation, au lieu du $16^{\mathrm{e}}$ au $20^{\mathrm{e}}$ jours seulement. La présente étude montre que cet effet est encore peu apparent à 60 jours et que c'est plus l'apport de protéines per se que la période durant laquelle il est effectué qui pourrait être en cause. L'écart de poids constaté à la naissance ne se manifeste pas significativement à 60 jours de gestation, alors que dans des conditions analogues, Atinmo ef al. (1976) notent une différence pondérale chez des fœtus âgés de 70 jours. Or, c'est précisément entre ces 2 dates qu'une compétition accrue intervient au niveau de la portée (Pomeroy, 1960) ; l'écart de 10 jours pourrait expliquer en partie les divergences entre les 2 études. L'absence d'une simple différence pondérale à 60 jours se retrouve également au niveau des mensurations faites sur certains organes, ou des résultats de composition chimique, tant des fotus que de leurs annexes, qui ne varient pas significativement entre les lots.

Inversement, les mêmes déterminations effectuées à la naissance indiquent que non seulement la croissance, mais aussi le développement des porcelets est affecté. 
Le poids du cœur, du foie, des reins, des surrénales, et la longueur du fémur, sont réduits dans les 3 lots protéiprives presque proportionnellement au poids des porcelets. Mais le poids du cerveau n'est pas influencé par la nutrition azotée de la mère. Atinmo et al. (1974b) constatent au contraire une réduction pondérale du cerveau, qui reste toutefois relativement moins importante que celle du reste de l'organisme. La composition chimique du placenta et des porcelets accuse une diminution de la teneur en matière sèche dans le lot $P P$ par rapport aux autres.

A la naissance le taux de protéines dans la matière sèche des porcelets du lot PP est abaissé, ce qui tendrait à prouver que le développement du tissu musculaire a été modifié par rapport à celui des autres tissus, adipeux en particulier. Ceci n'a pas été le cas dans les deux autres lots ayant reçu un régime équilibré pendant 25 jours et, à cet égard, on peut remarquer qu'en comparaison d'un apport de protéines pendant la phase de croissance rapide des fœtus (lot $P P$ ), une alimentation équilibrée fournie plus précocément (lot $\mathrm{Pp}$ ) a tout aussi bien permis un développement musculaire normal. Quoi qu'il en soit, la quantité de matières azotées fixées par les contenus utérins apparaît liée à la consommation de protéines de la truie et montre que le rôle de «tampon 》 classiquement attribué à la femelle gravide vis-à-vis de sa portée dans le cas d'une carence nutritionnelle devient insuffisant lorsque le déficił alimentaire est important, vraisemblablement en raison de l'épuisement des réserves protéiques mobilisables de la mère. Compte tenu des sacrifices de porcelets qui ont été effectués, leur taux de survie n'a pas été rapporté. Cependant, la différence de poids enregistrée à la naissance est accentuée au sevrage ; de plus, le nombre de portées parvenant à ce stade est moindre dans le lot carencé en protéines pendant toute la gestation, alors que celles des autres lots semblent épargnées. Le plus faible développement à la naissance des porcelets est certainement en cause, mais aussi la diminution de la quantité ou de la qualité du lait produit par les femelles ayant reçu un régime protéiprive en gestation, ainsi que Pond ef al., $(1968,1969)$ et De Geefer et al. (1973) l'onł mis en évidence.

\section{Conclusion.}

Cette étude confirme la possibilité de gestation pour des truies soumises à un régime à très faible teneur en protéines. Cependant, la distribution d'un tel aliment semble affecter le développement des fotus à partir de 60 jours d'âge et jusqu'à la parturition. Les réserves corporelles de la mère n'ont donc pas été suffisantes pendant cette période pour que leur mobilisation protège la portée d'une carence nutritionnelle sévère. On note toutefois que la consommation par la truie d'un régime équilibré durant une période assez courte en début ou fin de gestation a permis de limiter les conséquences néfastes précédentes en raison du rôle d'épargne ou de reconstitution des réserves mobilisables de cet apport chez la truie. Celle-ci ne semble pas manifester un besoin précis à aucun moment de la vie du fœtus. 


\section{Références}

ATINMO T., POND W. G., BARNES R. H., 1974a. Effect of diefary energy vs. protein restriction on blood constituents and reproductive performance in swine. J. Nutr., 104, 1033-1040.

ATINMO T., POND W. G., BARNES R. H., 1974b. Effect of maternal energy vs. protein restriction on growth and development of progeny in swine. J. anim. Sci., 39, 703-711.

ATINMO T., BALDIJAO C., POND W. G., BARNES R. H., 1976. Prenatal and postnatal protein malnutrition in pigs : effects on growth rate, serum protein and albumin. J. anim. Sci., 43, 606612.

DE GEETER M. J., HAYS V. W., KRATZER D. D., CROMWELL G. L., 1972. Reproductive performance of gilts fed diets low in protein during gestation and lactation. J. anim. Sci., 35, 772-777.

DE GEETER M. J., HAYS V. W., KRATZER D. D., CROMWELL G. L., 1973. Postweaning performance of pigs from gilts fed diets high and low in protein during gestation and lactation. J. anim. Sci., 37, 459-462.

DUÉE P. H., RÉRAT A., 1975 . Etude du besoin en lysine de la truie gestante nullipare. Ann. Zoofech., 24, 447-464.

DUÉE P. H., SĖVE B., 1978. Alimentation azotée du porc. Journées Rech. porcine en France, 10, INRAITP éd., 167-208.

ETIENNE M., HENRY Y., 1973. Influence de l'apport énergétique sur l'utilisation digestive et métabolique des nutriments, et les performances de reproduction, chez la truie gestante nullipare. Ann. Zootech., 22, 311-326.

HAMMELL D. L., KRATZER D. D., CROMWELL G. L., HAYS V. W., 1976. Effect of protein malnutrition of the sow on reproductive performance and on postnatal learning and performance of the offspring. J. anim. Sci., 43, 589-597.

POMEROY R. W., 1960. Infertility and neonatal mortality in the sow. IV. Further observations and conclusions. J. agric. Sci., 54, 57-66.

POND W. G., WAGNER W. C., DUNN J. A., WALKER E. F. Jr., 1968. Reproduction and early postnatal growth of progeny in swine fed a protein-free diet during gestation. J. Nufr., 94, 309-316.

POND W. G., STRACHAN D. N., SINHA Y. N., WALKER E. F. Jr., DUNN J. A., BARNES R. H., 1969. Effect of protein deprivation of swine during all or part of gestation on birth weight, postnatal growth rate and nucleic acid content of brain and muscle of progeny. J. Nutr., 99, 61-67.

RIPPEL R. H., RASMUSSEN O. G., JENSEN A. H., NORTON H. W., BECKER D. E., 1965. Effect of level and source of protein on reproductive performance of swine. J. anim. Sci., 24, 203-208.

SALMON-LEGAGNEUR E., 1964. Etude du besoin qualitatif d'azole chez la truie en gestation et en lactation. Comparaison de deux protéines : farine de poisson et arachide. Ann. Zoofech., 13, 51-61. 